\title{
Separation science of macromolecules
}

\author{
André M. Striegel
}

Published online: 17 December 2010

(C) Springer-Verlag 2010

In 1973, Bruno Vollmert wrote "The only completely satisfactory description of the molecular weight (i.e., the degree of polymerization) of a macromolecular compound is the distribution curve... as determined through fractionation" [1]. The accuracy of this statement stems, in large part, from two facts: First, that the molar mass (formerly known as molecular weight) distribution provides information on properties such as the elongation and tensile strength of materials. Second, that while the various molar mass averages $\left(M_{n}, M_{w}, M_{z}\right.$, etc.) of a polymer provide important information on their own, it is quite possible for macromolecules with vastly different molar mass distributions to have the exact same molar mass averages and polydispersities. This is true of any statistical distribution and, as such, Vollmert's statement applies equally well to the distribution of any macromolecular property, of which there are many: Long and short-chain branching, chemical composition, tactility, sequence length, polyelectrolytic charge, etc.

Over the last half-century, size-exclusion chromatography (SEC) has established itself as the premier method for obtaining the molar mass distribution (MMD) of natural and synthetic polymers. Enhanced by a multiplicity of physicochemical detection methods, it can also be used to quantify many of the heterogeneities present in macromolecules and increase our knowledge of polymer architecture and dilute solution thermodynamics. The technique

Published in the special issue on Separation Science of Macromolecules with Guest Editor André Striegel.

A. M. Striegel $(\triangle)$

Department of Chemistry \& Biochemistry, Florida State

University,

Tallahassee, FL 32306-4390, USA

e-mail: striegel@chem.fsu.edu is not without its limitations, however. First, it should always be remembered that, by definition, SEC is a technique that separates analytes on the basis of their size in solution, not necessarily on the basis of analyte molar mass. A consequence of this is the phenomenon of local polydispersity, in which molecules which differ from each other in architecture, chemical composition, or both may co-elute from the SEC column because of the similar hydrodynamic volumes occupied by these molecules. Although it may be possible to address this problem by use of multiple physical detectors, this requires highly accurate band-broadening corrections and interdetector delay calculations and, quite often, for the co-eluting species to have specific refractive index increments that are extremely different from each other. Another approach to the problem of local polydispersity has been to circumvent it altogether. This is seen in the review by Gilbert who, rather than determining the MMD of heterogeneously branched polysaccharides for which local architectural polydispersity might be observed in size-based separations, instead examines the size distribution of the analytes to derive conclusions therefrom.

Another limitation of SEC stems from the large shear rates to which macromolecules are exposed during their passage through the packed, porous medium of the chromatographic column. The ease with which large polymers may degrade under even the mildest of SEC conditions (employing ultra-low flow rates and columns packed with large-diameter particles) has encouraged the development and use of gentler, alternative size-based techniques. Chief among the latter is the family of methods known as field-flow fractionation (FFF) and, in particular, flow FFF, also known as A4F. As demonstrated by a multiplicity of papers in this special issue, A4F has found favor in the characterization of biopolymers, especially 
polysaccharides. A second size-based technique which is currently experiencing a resurgence, aided by the power of multiple detection, is hydrodynamic chromatography (HDC). While the resolution in HDC is usually lower than in FFF, the former gains from its ability to use the same instrumental setup as employed for SEC experiments, with only a different set of columns needed. In this special issue, HDC is discussed in the paper by Rolland-Sabaté and colleagues, who demonstrate the complementarity of the technique to SEC and $\mathrm{A} 4 \mathrm{~F}$ in the analysis of native starches, and in the paper by Brewer and Striegel, who use quadruple-detector HDC to characterize the size, shape, and compactness of a synthetic drug-delivery vesicle.

Although multi-detector SEC, HDC, and FFF can provide information on the molar mass and size distributions of macromolecules and on a variety of physical and chemical heterogeneities present, these techniques tell us nothing about the other types of distributions that may coexist along with the MMD. To this effect, we benefit from the application of so-called "interactive" techniques such as liquid adsorption chromatography, temperature rising elution and crystallization fractionation (TREF and CRYSTAF, respectively), gradient polymer elution chromatography, etc. The first set of methods is covered in the manuscripts by Trathnigg and by Macko et al. who use the methods, respectively, to quantify the distribution of poly(ethylene glycols) and their monomethyl ethers in samples and for the separation of short-chain branched polyolefins. The second set of methods is represented in the paper by Monrabal and del Hierro, who use TREF and CRYSTAF to characterize blends of polypropylene and polyethylene.

Microfluidic analysis is an area that has met with much success in the bioanalytical community and which has great promise for the analysis of macromolecules and particles. In this special issue, two very different applications of microfluidics to macromolecular separation science are presented: Janča describes the relaxation of microparticles in microfluidic conduits whereas Baker et al. compare the performance of laser-ablated and wet-etched microfluidic devices in the separation of both large and small biomolecules. This is an area in which we are sure to see great advances. Further advances in instrumentation and novel couplings are seen in the paper by Yau and colleagues, who introduce a novel type of on-line viscometer, and in the paper by Watanabe and Inoko, who show how SEC can be coupled on-line to smallangle X-ray scattering for analysis of a hollow sphere protein complex and of a linear polysaccharide.
Every year, the production of natural and synthetic macromolecules grows, fueled chiefly by the demand for newer and better polymer-based products. To help optimize the design, processing, and end-use of these materials in non-empirical fashion, through better understanding of the macromolecules and their structure-property relationships, is the objective of macromolecular separation science. The original papers, reviews, and technical notes in this special issue of Analytical and Bioanalytical Chemistry report the state-of-the-art of many size-based and interactive macromolecular separation methods, thus laying the groundwork for future advances in this important area that lies at the intersection of analytical chemistry, polymer and colloidal science, biochemistry, and carbohydrate and food science, among others. As Guest Editor, I thank all the authors for sharing their expertise and knowledge and also extend this gratitude to the anonymous reviewers who ensured the quality and clarity of the work presented. Last, I thank the editorial staff of the journal, who have made my job substantially easier than it would have been without them.

\section{Reference}

1. Vollmert B (1973) Polymer chemistry. Springer-Verlag, New York

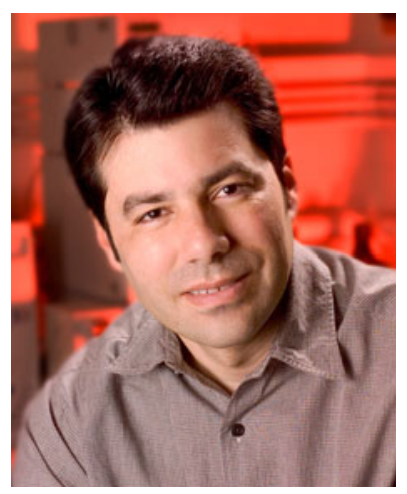

André M. Striegel is Assistant Professor in the Department of Chemistry and Biochemistry at Florida State University (FSU), where his group's research focuses on the separation science of macromolecules. Dr Striegel is the author of nearly 50 peerreviewed papers, lead co-author of the second edition of the book "Modern Size-Exclusion Liquid Chromatography" (2009), and editor of the book "Multiple Detection in SizeExclusion Chromatography" (2005). He has served as inaugural Professor in Residence for Preservation Research and Testing at the US Library of Congress. Dr Striegel was the inaugural recipient of the ACS-DAC Award for Young Investigators in Separation Science, and has also received the Eli Lilly Analytical Chemistry Grantee Award, an FSU First Year Assistant Professor Award, and a Solutia Technical Achievement Award for his research in private industry before coming to academia. He has served on the editorial board of Analytical and Bioanalytical Chemistry since 2008 and, since 2009, as associate editor of the Encyclopedia of Analytical Chemistry. 